\title{
Determination of 15 phthalate esters by ultra performance convergence chromatography
}

\author{
Wulin $\mathrm{Li}^{\mathrm{a}}$, Genrong $\mathrm{Li}^{\mathrm{b}}$, Xiao Zou ${ }^{\mathrm{c}}$, Lingling, Zhu ${ }^{\mathrm{d}}$, Jiali $\mathrm{Lu}^{\mathrm{e}}$ and Yanfei $\mathrm{Li}^{\dagger}$ \\ Chongqing Academy of Metrology and Quality Inspection, Chongqing 401123, China \\ alw1656pll@163.com, b734253977@qq.com, ${ }^{\text {c}}$ cutie621@163.com, d1447826865@qq.com, ${ }^{\text {e}} 312986$ \\ 913@qq.com, flyf@cqjz.com.cn
}

Keywords: ultra performance convergence chromatography $\left(\mathrm{UPC}^{2}\right)$, photodiode array detector (PDA), phthalate esters, determination

\begin{abstract}
Ultra Performance convergence chromatography (UPC ${ }^{2}$ ) is cost effective, sustainable, and uses green technology that lowers the use of organic solvents. Based on this advantage, a method was developed for the determination of 15 phthalate esters by UPC ${ }^{2}$. The separation of 15 phthalate esters was achieved on an ACQUITY UPC ${ }^{2}$ HSS C18 SB column $(150 \mathrm{~mm} \times 3 \mathrm{~mm}, 1.8 \mu \mathrm{m})$ by a gradient elution with supercritical $\mathrm{CO}_{2}$ and acetonitrile as mobile phases. External standard method was used for the quantitative determination and the calibration curves showed good linearity in the concentration range of $0.5-10 \mathrm{mg} / \mathrm{L}$ with correlation coefficients varying from 0.9962 to 0.9998 . The limits of detection were $0.05 \sim 0.11 \mathrm{mg} / \mathrm{L}$. The method could be successfully applied for the determination of the phthalate esters in plastic products etc.
\end{abstract}

\section{Introduction}

Phthalates esters (PAEs) are widely used as plasticizers and additives in many daily products such as plastics, pesticides, paints and cosmetics [1]. PAEs can easily release and migrate from products and migrate into food or water that comes into direct contact. Because PAEs have carcinogenic and estrogenic impact on human health, a maximum admissible concentration for specific PAEs had established.

The most commonly used techniques for analyzing PAEs consist of: gas chromatography coupled with electron capture, flame ionization, mass spectrometry (MS) detection and high performance liquid chromatography (HPLC) coupled with ultraviolet (UV) and MS detection[2-4].

Ultra-performance convergence chromatography (UPC ${ }^{2}$ ) integrates supercritical fluid chromatography (SFC) and UPLC technologies, and shows many remarkable advantages including cost effective, sustainable, and uses green technology that lowers the use of organic solvents [5]. To date, it has not been applied in separating PAEs. In this work, a sensitive UPC ${ }^{2}$ method was established to simultaneously determine 15 PAEs.

\section{Materials and methods}

\subsection{Chemicals.}

Methanol, 2-propanol, acetonitrile and hexane were of HPLC grade and purchased from Tedia Company, Inc (USA). Reference standards of Diisohexyl phthalate(BMPP), diisobutyl phthalate(DIBP), dibutyl phthalate(DBP), diethyl phthalate(DEP), diethylexyl phthalate(DEHP), Dimethyl phthalate(DMP), Di-n-hexyl phthalate(DHXP), Di(n-octyl) phthalate(DNOP), Butyl benzyl phthalate(BBP), Diphenyl Phthalate(DPhP), Diisononyl phthalate(DINP), Dicyclohexyl phthalate(DCHP), Bis(2-butoxyethyl) phthalate(DBEP), Bis(2-Butoxyethyl) Phthalate(DEEP) and Dimethoxyethyl phthalate(DMEP) were obtained from Beijing haianhongmeng Reference Material Technology Co, Ltd. 


\subsection{Experiment conditions.}

PAEs analysis was performed using an ACQUITY UPC ${ }^{2}$ system with photodiode array detector (Waters, USA). Methanol, methanol/ 2-propanol (1:1) were used as strong and weak needle wash solvents. The separation was performed using four ACQUITY UPC ${ }^{2}$ columns, BEH(100 mm $\times 3 \mathrm{~mm}$, 1.7 $\mu \mathrm{m})$, BEH 2-EP(100 $\mathrm{mm} \times 3 \mathrm{~mm}, 1.7 \mu \mathrm{m})$, CSH Fluoro-Phenyl $(100 \mathrm{~mm} \times 3 \mathrm{~mm}, 1.7 \mu \mathrm{m})$ and HSS C18 SB(150 mm $\times 3 \mathrm{~mm}, 1.8 \mu \mathrm{m})$. Gradient elution was performed using $\mathrm{CO}_{2}$ ( $>99.97 \%$ of purity ) and various modifiers including methanol, 2-propanol and acetonitrile at flow-rate $1.5 \mathrm{ml} / \mathrm{min}$. UV detection was performed at $220 \mathrm{~nm}$.

\subsection{Sample preparation.}

Standard stock solutions were prepared by diluting each compound with hexane. The stock solutions were further diluted to $0.5,1.0,2.0,5.0,10 \mathrm{mg} / \mathrm{L}$ in order to obtain calibration curves. mixed standard solutions were stored at $4^{\circ} \mathrm{C}$ until use.

\section{Results and Discussions}

\subsection{The influence of stationary phase}
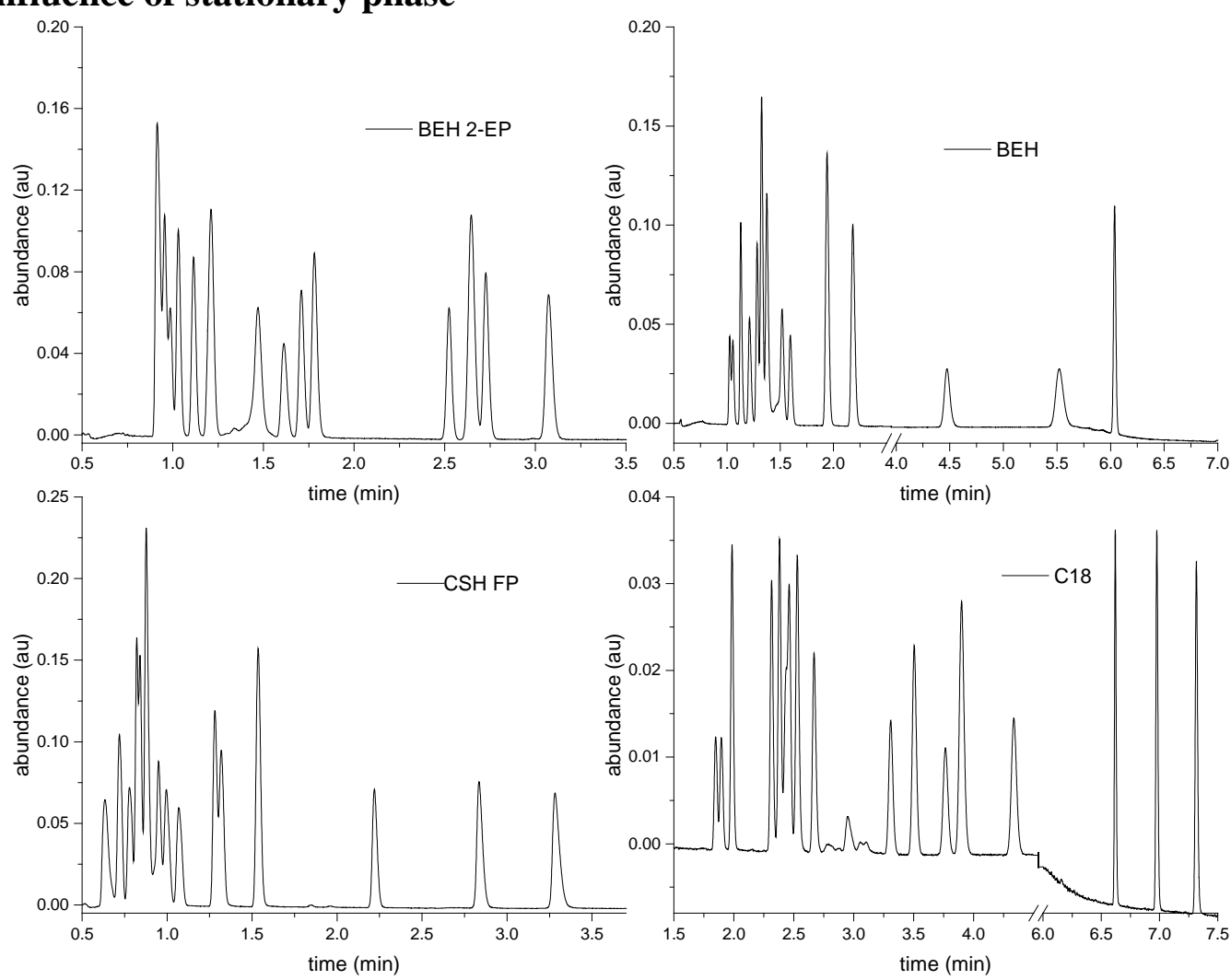

Fig. 1 Effect of different stationary phase on the separation of 15 PAEs

Stationary phase has the strongest impact on the selectivity of separation. These stationary phases differed both in selectivity and polarity representing very polar (BEH and BEH 2-EP), moderately polar (CSH Fluoro-Phenyl) and non-polar (HSS C18 SB) stationary phases. Fig. 1 shows chromatograms using the section 2.1 mentioned columns with the same composition of mobile phase. Compared to other stationary phases, the HSS C18 SB column resulted in better resolution and peak shape.

\subsection{The influence of modifier solutions}

Modifiers solutions are usually added to supercritical fluids $\mathrm{CO}_{2}$ to change eluent strength of the mobile phase and to improve peak shape. Fig. 2 shows that HSS C18 SB column separated the PAEs with three modifiers (methanol, acetonitrile and 2-propanol). Methanol and 2-propanol failed to separate targets peaks though it significantly reduced retention times, which no longer eluted after 2 
min. Acetonitrile gave the best results wherein 15 peaks could be readily observed even though peaks DBP, DEP, DEHP, DMP and DHXP were not fully resolved.

\subsection{The optimal conditions}

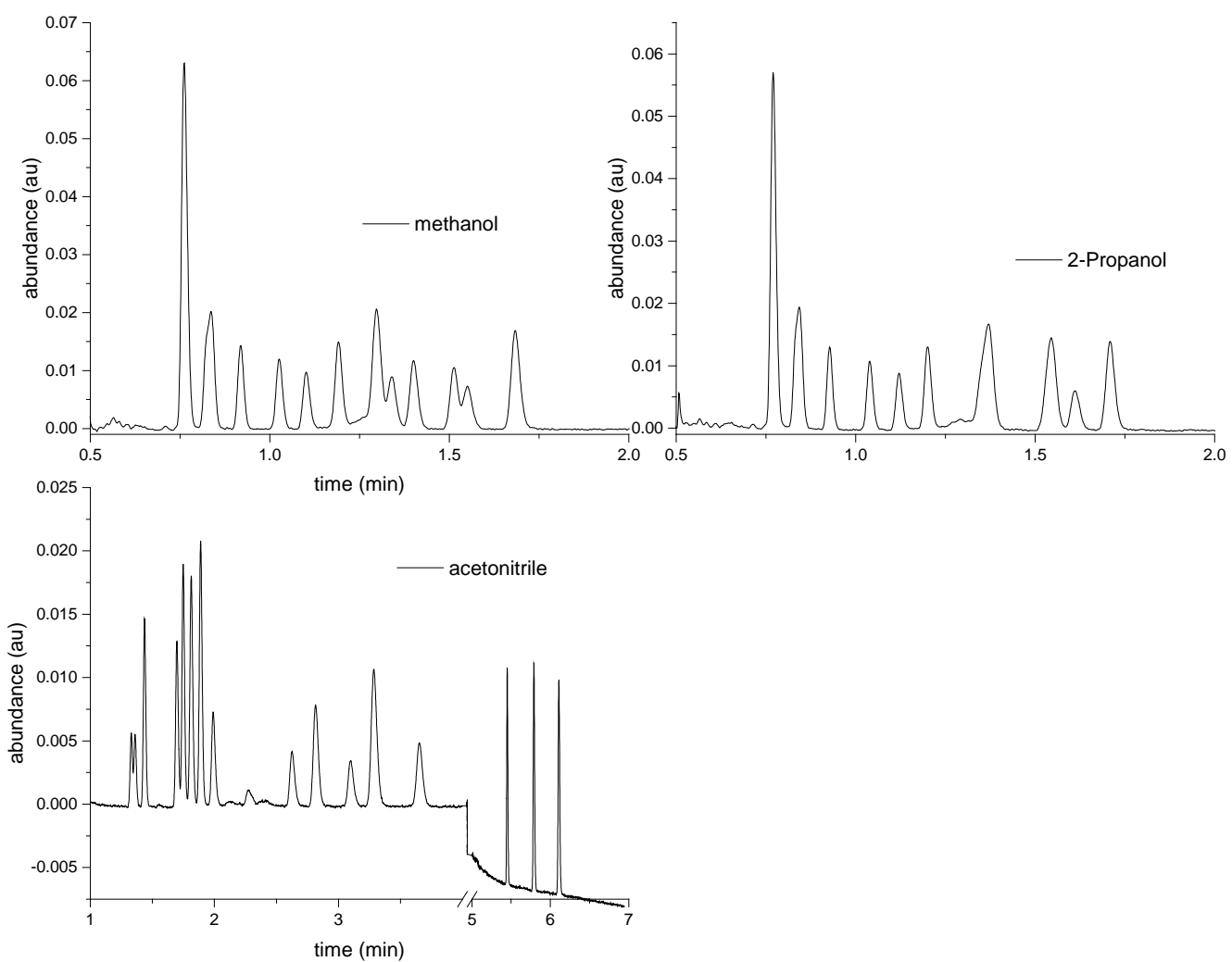

Fig. 2 Effect of different solutions on the separation of 15 PAEs

Based on evaluation of flow rate, column temperature, and back pressure, the optimal conditions were obtained: standard elution gradient program of acetonitrile (B) in $\mathrm{CO}_{2}$ (A), 3\%B(initial), 3-5\% B (0-2min), 5\% B(2-5min), 5-15\%B(5-5.5min), 15-20\%B (5.5-7.5min), 20-3\%B(7.5-8 min). The back pressure was set at 1800 psi. The flow rate was $1.5 \mathrm{~mL} / \mathrm{min}$ while the injection volume was $3 \mu \mathrm{L}$. The column temperature was maintained at $65^{\circ} \mathrm{C}$. UV detection was performed at 220nm. Chromatogram of 15 PAEs obtained at optimal conditions were showed as Fig. 3.

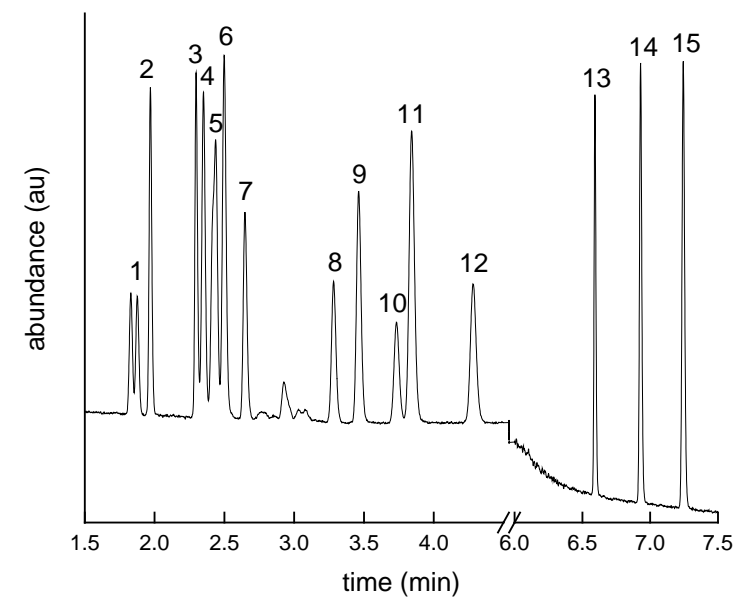

Fig. 3 Chromatogram of a mixture of the 15 PAEs

\subsection{Method validation}

Quantitative analysis of 15 PAEs was performed at optimized conditions as described in Section 3.3. Table 1 displays the results obtained for the Regression equation, Correlation coefficients and LODs. The linearity obtained for each of the calibration curves was satisfactory with correlation coefficients 
$\left(\mathrm{R}^{2}\right.$ ) ranging from 0.9960 to0.9999. Limits of detection (LODs) were calculated at signal-to-noise ratio of 3, and the calculated LODs of 15 PAEs were $0.05 \sim 0.11 \mathrm{mg} / \mathrm{L}$.

Table 1 Retention time, Regression equation, $\mathrm{R}^{2}$ and LODs of 15 PAEs

\begin{tabular}{cccccc}
\hline No. & Compound & Retention time $(\mathrm{min})$ & Regression equation & $\mathrm{R}^{2}$ & LOD $(\mathrm{mg} / \mathrm{L})$ \\
\hline 1 & BMPP & 1.848 & $\mathrm{Y}=1350 \mathrm{X}+70.8$ & 0.9995 & 0.06 \\
2 & 1.897 & $\mathrm{Y}=1360 \mathrm{X}+388$ & 0.9998 & 0.11 \\
3 & DIBP & 1.985 & $\mathrm{Y}=3190 \mathrm{X}+341$ & 0.9990 & 0.08 \\
4 & DBP & 2.316 & $\mathrm{Y}=3090 \mathrm{X}+379$ & 0.9994 & 0.09 \\
5 & DEP & 2.382 & $\mathrm{Y}=3940 \mathrm{X}+109$ & 0.9992 & 0.07 \\
6 & DEHP & 2.462 & $\mathrm{Y}=5190 \mathrm{X}+213$ & 0.9994 & 0.08 \\
7 & DMP & 2.530 & $\mathrm{Y}=4570 \mathrm{X}+299$ & 0.9994 & 0.05 \\
8 & DHXP & 2.669 & $\mathrm{Y}=2720 \mathrm{X}+183$ & 0.9996 & 0.05 \\
9 & DNOP & 3.309 & $\mathrm{Y}=2180 \mathrm{X}+183$ & 0.9997 & 0.09 \\
10 & BBP & 3.505 & $\mathrm{Y}=3370 \mathrm{X}+347$ & 0.9971 & 0.07 \\
11 & DINP & 3.765 & $\mathrm{Y}=2170 \mathrm{X}+79.2$ & 0.9992 & 0.11 \\
12 & DPhP & 3.900 & $\mathrm{Y}=4750 \mathrm{X}-366$ & 0.9996 & 0.08 \\
13 & DCHP & 4.335 & $\mathrm{Y}=2790 \mathrm{X}+579$ & 0.9962 & 0.07 \\
14 & DBEP & 6.620 & $\mathrm{Y}=2500 \mathrm{X}+299$ & 0.9992 & 0.10 \\
15 & DEEP & 6.977 & $\mathrm{Y}=3040 \mathrm{X}+403$ & 0.9988 & 0.07 \\
\hline
\end{tabular}

Y: peak area; X: mass concentration, $\mathrm{mg} / \mathrm{L}$

\section{Summary}

In this study, a method for the determination of 15 PAEs utilizing UPC ${ }^{2}$ coupled with PDA was established. Under the optimal conditions, the calibration curves showed good linearity in the concentration range of $0.5-10 \mathrm{mg} / \mathrm{L}$ for 15 PAEs with correlation coefficients varying from 0.9962 to 0.9998. The limits of detection were $0.05 \sim 0.11 \mathrm{mg} / \mathrm{L}$. The method could be applied for the determination of PAEs in plastic products etc.

\section{References}

[1]. Suzuki Y, Yoshinaga J, Mizumoto Y, et al. Foetal exposure to phthalate esters and anogenital distance in male newborns. International journal of andrology, Vol. 35(2012), No. 3, p. 236-244.

[2]. Wu P G, Pan X D, Ma B J, et al. Determination of phthalate esters in non-alcoholic beverages by GC-MS and optimization of the extraction conditions. European Food Research and Technology, Vol. 238(2014), No. (4), p. 607-612.

[3]. Chen B, Zhang L. An easy and sensitive analytical method of determination of phthalate esters in children's toys by UPLCMS/MS. Polymer Testing, Vol. 32(2013), No. 4, p. 681-685.

[4]. Wu Q, Liu M, Ma X, et al. Extraction of phthalate esters from water and beverages using a graphene-based magnetic nanocomposite prior to their determination by HPLC. Microchimica Acta, Vol. 177(2012), No. (1-2), p. 23-30. 
[5]. Gong X, Qi N, Wang X, et al. Ultra-performance convergence chromatography (UPC ${ }^{2}$ ) method for the analysis of biogenic amines in fermented foods. Food chemistry, Vol. 162(2014), p. 172-175. 